\title{
X-RAY SPECTROSCOPIC CHARACTERIZATION OF SHOCK-IGNITION-RELEVANT PLASMAS
}

\author{
Michal ŠmíD $^{a, b, *}$, Luca Antonelli ${ }^{c, d}$, Oldřich RenneR ${ }^{a}$ \\ ${ }^{a}$ Institute of Physics of the ASCR, v.v.i., Prague, Czech Republic \\ ${ }^{b}$ Czech Technical University in Prague, FNSPE, Prague, Czech Republic \\ c Dipartimento di Ingegneria Industriale, Universita Roma 'Tor Vergata', Roma, Italy \\ ${ }^{d}$ CELIA, Université de Bordeaux 1, Talence, Bordeaux, France \\ * corresponding author: smidm@fzu.cz
}

\begin{abstract}
Experiments with multilayer plastic/Cu targets performed at a PALS laser system aimed at the study of matter at conditions relevant to a shock ignition ICF scheme, and, in particular, at the investigation of hot electrons generation. Plasma temperature and density were obtained using high-resolution X-ray spectroscopy. 2D-spatially resolved quasi-monochromatic imaging was observing the hot electrons via fluorescence $\mathrm{K} \alpha$ emission in the copper tracer layer. Found values of plasma temperature $690 \pm 10 \mathrm{eV}$, electron density $3 \times 10^{22} \mathrm{~cm}^{-3}$ and the effective energy of hot electrons $45 \pm 20 \mathrm{keV}$ demonstrate the potential of X-ray methods in the characterization of the shock ignition environmental conditions.
\end{abstract}

KEYWORDS: hot electrons, shock ignition, laser-produced plasma, X-ray spectroscopy, K $\alpha$ radiation.

\section{INTRODUCTION}

Shock ignition (SI) scheme is one of the alternative approaches to the inertial confinement fusion. It anticipates a compression of the spherical target by a laser beam with intensity $\approx 1 \times 10^{14} \mathrm{~W} \mathrm{~cm}^{-2}$, followed by an intense pulse $\left(\approx 1 \times 10^{16} \mathrm{~W} \mathrm{~cm}^{-2}\right)$ which drives the strong converging igniting shock with pressures at the ablation front up to several 100 Mbar [2].

Under these conditions, the igniting (main) beam is not absorbed in the solid target, but interacts with the underdense plasma corona, where parametric instabilities take place. Primarily, the stimulated Raman scattering occurring below the $n_{\mathrm{c}} / 4$ limit is a significant mechanism of collisionless absorption. In this process, a plasma wave is excited along the laser direction and a light beam is reflected with a modified frequency spectrum. The plasma wave accelerates free electrons to suprathermal energies.

These 'hot' electrons may affect the SI target significantly, but their role is not fully understood yet: they can either preheat the compressed material, which would lead to premature target expansion and the target gain decrease, or they may be stopped in the dense shell of the target, thus increasing the ablation pressure, improving the symmetry of the converging shock pressure front and increasing the gain [10].

The aim of the experiments performed in the PALS research center [6] was to create plasma with conditions relevant for the SI and implement advanced spectroscopic diagnosis to validate the this relevance. The collected data should contribute to the investigation of hot electrons generation and propagation in dense target.

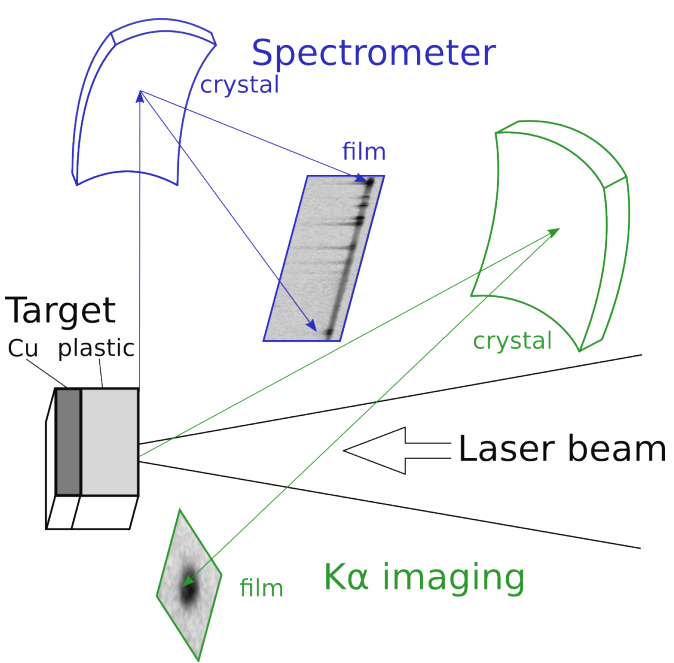

Figure 1. Experimental setup.

\section{EXPERIMENTAL SETUP}

The scheme of the experiment shown in Fig. 1 includes the multi-layer target, the irradiating laser beam, the X-ray spectrometer, and the $\mathrm{K} \alpha$ imaging system.

The target consisted of a 25 or $40 \mu \mathrm{m}$ thick layer of chlorine-doped plastic (parylene-C; $\mathrm{C}_{8} \mathrm{H}_{7} \mathrm{Cl}$ ) and a $5 \mu \mathrm{m}$ thick copper tracer layer. The relevance of using doped plastic targets as an SI ablator layer has been discussed, e.g., in paper [3, similar measurements have also been performed [4].

The target was irradiated from the plastic side. The prepulse $(1315 \mathrm{~nm}, 70 \mathrm{~J}$, focal spot diameter $700 \mu \mathrm{m}, \approx 6 \times 10^{13} \mathrm{~W} \mathrm{~cm}^{-2}$ ) generates a long-scale 
preplasma corresponding to the compression phase of the SI. The frequency-tripled PALS main beam ( $438 \mathrm{~nm}, 170 \mathrm{~J}$, focal spot diameter $80 \mu \mathrm{m}$, pulse length $300 \mathrm{ps}, \approx 1 \times 10^{16} \mathrm{~W} \mathrm{~cm}^{-2}$ ) strikes the target at a variable delay, generating a shock wave and the hot electrons. These electrons propagate into the copper layer and create vacancies in the $\mathrm{K}$-shell of the $\mathrm{Cu}$ atoms which consequently emit the fluorescence $\mathrm{K} \alpha$ radiation.

The X-ray spectra were measured using a spherically-bent mica crystal spectrometer which was aligned to provide a spatial resolution along the laser axis. In the $4^{\text {th }}$ order, it covered the wavelengths $4.17 \div 4.52 \AA$ to image the $\mathrm{Cl} \mathrm{He}_{\alpha}$ and $\mathrm{Ly}_{\alpha}$ lines. The $\mathrm{He}_{\gamma} \div \mathrm{He}_{\eta}$ and $\mathrm{Ly}_{\beta}$ lines were observed in the $5^{\text {th }}$ order.

The $\mathrm{Cu} \mathrm{K} \alpha$ emission was measured using a spherically-bent quartz (211) crystal, which was set up as a monochromator in imaging mode (Bragg angle $\theta=88.7^{\circ}$ ) to provide a quasi-monochromatic distribution of $\mathrm{K} \alpha$ intensity, 2D-spatially resolved along the target surface.

Both diagnostics used the Kodak AA400 film to detect the signal.

\section{X-RAY SPECTRA EVALUATION}

The measured spectral records were digitized using a calibrated table-top scanner and recalculated to optical densities. The raw record with lines identification is in Fig. 2, This record was split into regions corresponding to the $4^{\text {th }}$ and $5^{\text {th }}$ spectroscopic orders, each recalculated into emitted intensity using theoretical energy-dependent reflectivity, filter transmission, and film response.

The dominant $\mathrm{Ly}_{\beta}$ and $\mathrm{He}_{\delta}$ lines were selected for the evaluation. The ratio of these two lines, reflecting the ratio of hydrogen- and helium-like ions, is very sensitive to plasma temperature, while their width is given mainly by the Stark broadening thus providing a very sensitive tool for plasma density estimation. Though their recorded intensities are comparable to the $\alpha$ lines, their emissivities are relatively low. This is because the crystal reflectivity and filter transmission are increasing with the decreasing wavelength. The low optical thickness of these lines is very beneficial for the diagnostic purposes, as it minimizes undesirable effects like opacity broadening or reabsorption in inhomogeneous plasma.

The temperature and density were determined from the best fit of the observed spectra with simulations. A set of needed spectra with variable parameters was generated by using the PrismSpect code [8] under the assumption of homogeneous planar plasma and a steady-state approximation.

The fitting was done using the least square method. The logarithm of the least squares of differences between the experimental and synthetic spectra for a typical experimental data, shown as a function of the temperature and density of the theoretical spectra, is plot-

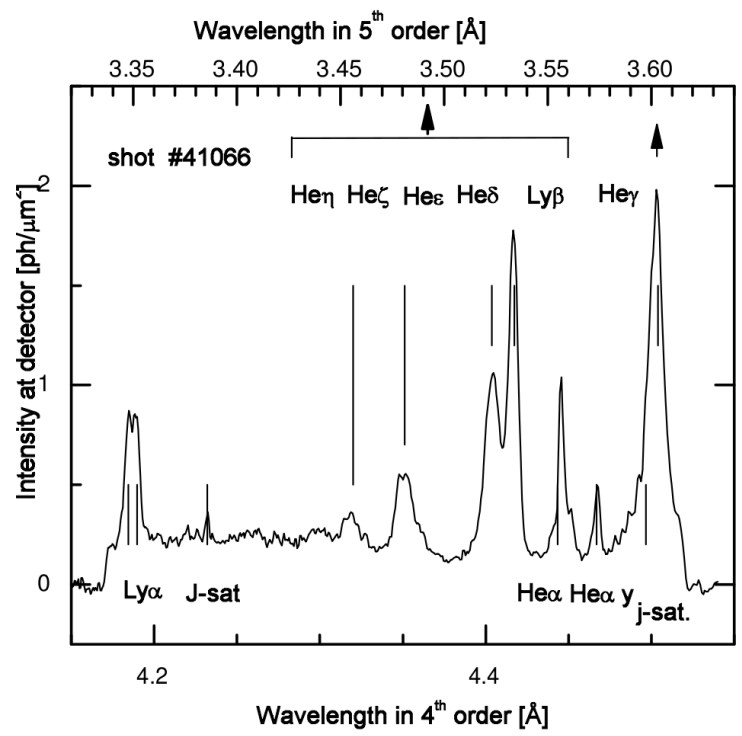

FIgURE 2. Raw spectral record with lines identification.

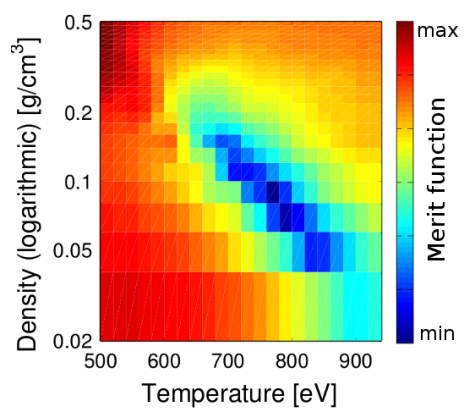

Figure 3. The dependence of the merit function on $T$ and $\rho$; the diagonally-elongated minimum indicates the uncertainty of the plasma parameters estimation.

ted in Fig. 3. This figure characterizes the uncertainty of the parameter estimation: the diagonallyelongated minimum represents a set of synthetic spectra with a good agreement to the experimental one. To increase the precision of the plasma parameters estimation, the density has been separately derived using the FWHM width of the $\mathrm{Ly}_{\beta}$ line. Having the density fixed, the uncertainty of the temperature estimation was about $20 \mathrm{eV}$.

Figure 4 shows the comparison of the reconstructed experimental spectrum with the best fitting theoretical one; the $5^{\text {th }}$ order lines are magnified in the inset.

\section{4. $\mathrm{K} \propto$ EVALUATION}

The $2 \mathrm{D}$-spatially resolved $\mathrm{K} \alpha$ record directly provides the information on radius and intensity of the $K \alpha$ signal. This data was used to evaluate the hot electrons effective energy and absolute population.

\subsection{EFFECTIVE ENERGY}

The effective energy of the hot electron beam was estimated by analyzing the attenuation of the signal in dependence on the plastic layer thickness. As the experiments were conducted with various thicknesses, 


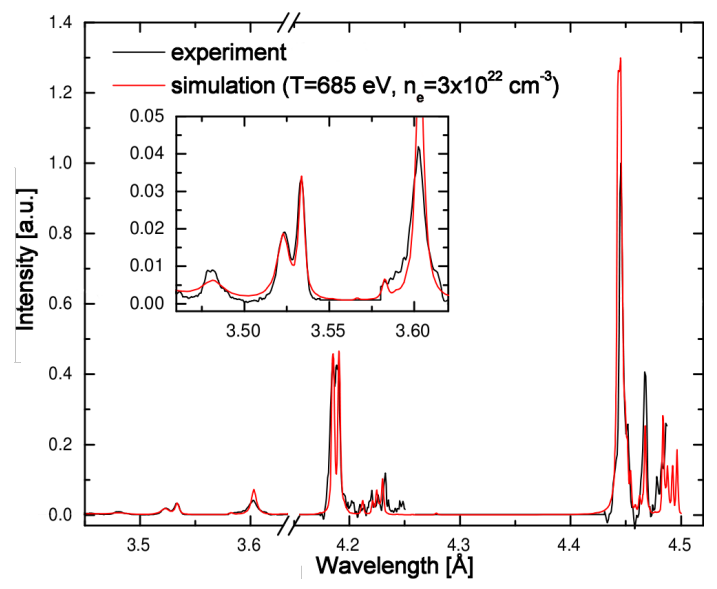

Figure 4. Comparison of the reconstructed experimental spectrum and the best fitting theoretical one.

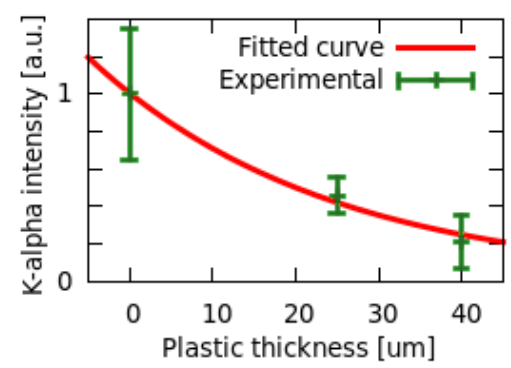

FiguRE 5. The dependence of the normalized integrated $K \alpha$ intensity on the plastic layer thickness, its experimental values and fitted attenuation curve.

namely 0,25 and $40 \mu \mathrm{m}$, the signal intensity could be plotted as a function of this thickness and fitted with an assumed exponential attenuation (Fig. 5). Comparing the attenuation with the stopping power from the Estar database [1], the mean electron energy was found to be about $45 \pm 20 \mathrm{keV}$.

This high uncertainty is caused mainly by the hot electron production process. In the relevant intensity regime, hot electrons are generated by strongly nonlinear processes (stimulated Raman scattering and two plasmon decay) which are correlated to the stability of the laser system and laser-plasma interaction. As a consequence, the energy of hot electrons can vary a lot between comparable shots. For our purposes, the essential information is the order of magnitude of this energy which equals to tens of $\mathrm{keV}$.

\subsection{Absolute Calibration}

To perform the absolute calibration of the measured data, it is necessary to relate the detected signal to the $\mathrm{K} \alpha$ emission, and to relate this $\mathrm{K} \alpha$ emission to the number of hot electrons propagating through the copper layer.

The first problem was solved using a detailed quantitative analysis based on a ray-tracing procedure. The calculations follow the standard algorithms described, e.g., in paper [11]. The simulation assumes a point source giving an origin to a fan of isotropic quasi monochromatic X-rays, each ray carries an equivalent part of the emitted intensity. By default, it is assumed that the source is emitting 1 photon into the full solid angle. The reflection curve of the spherically bent crystal is calculated by using the modified Taupin equation [5]. By taking into account all relevant geometric factors (source-to-crystal and crystal-todetector distances, crystal and detector dimensions), the directions and amplitudes of the rays reflected from the crystal are found and the signal distribution at the detector plane is calculated. The collection efficiency is then determined by integrating the signal over the active detector area. The resulting ratio of the detected to emitted radiation is only $2.4 \times 10^{-6}$, which is more than one order of magnitude less than might be expected when neglecting the variation of the incidence angle. The full number of the emitted $K \alpha$ photons is determined with respect to the transmission of the protective filters and the characteristic curve of the X-ray film used.

To relate the number of hot electrons $\left(n_{\mathrm{HE}}\right)$ to the number of $\mathrm{K} \alpha$ photons $\left(n_{\mathrm{K} \alpha}\right)$ emitted, the assumption of monoenergetic $50 \mathrm{keV}$ electron beam was made. The collisional cross section for $\mathrm{Cu}$ K-shell ionization is $\sigma=4 \times 10^{-22} \mathrm{~cm}^{-2}[9]$. Using the thin target approximation, the number of excited copper ions is

$$
n_{\mathrm{Cu} *}=\sigma d n_{\mathrm{Cu}} n_{\mathrm{HE}},
$$

where $n_{\mathrm{Cu}}$ is the number density of copper atoms. The probability that the excited copper ion will decay through radiative recombination is $W_{k}=0.39$ [7] and the final relation is

$$
n_{\mathrm{K} \alpha}=\sigma d n_{\mathrm{Cu}} W_{k} n_{\mathrm{HE}} .
$$

Using this formula, the absolute number of hot electrons propagating through the copper layer can be estimated.

\section{RESUlts AND DISCUSSION}

Several shots with similar conditions and with variable delays between the prepulse and the main beam have been analysed. The measured parameters did not show any dependence on the delay, so the impact of the prepulse in this situation can be considered as negligible.

The found plasma temperature was $T=690 \pm 10 \mathrm{eV}$, and the density $\rho=0.10 \pm 0.02 \mathrm{~g} \mathrm{~cm}^{-3}$ (which corresponds to $\left.n_{\mathrm{e}}=3 \times 10^{22} \mathrm{~cm}^{-3}\right)$.

The total number of $\mathrm{Cu} \mathrm{K} \alpha$ photons emitted was in the range $3 \div 30 \times 10^{9}$, which corresponds to the production of hot electrons $n_{\mathrm{HE}}=2 \div 20 \times 10^{11}$. Since this number is lower than theoretical predictions, further experiments are planned to revise this measurement in the future. The important value measured is the effective energy of hot electrons, estimated as $45 \pm 20 \mathrm{keV}$. 


\section{Conclusion}

High-resolution X-ray spectroscopy combined with monochromatic $\mathrm{K} \alpha$ imaging was implemented in laserplasma interaction experiments with laser intensities $\approx 1 \times 10^{16} \mathrm{~W} \mathrm{~cm}^{-2}$ and with weaker prepulse using variable delay to the main beam, thus in this matter relevant for the shock-ignition ICF scheme. The usability of this diagnostics was demonstrated, and sample data needed for the explanation of hot electrons generation, which is decisive for the success of the shockignition scheme, were collected. The measured data did not exhibit any distinct dependence on the prepulse timing. There are several alternate scenarios explaining this unexpected behavior, their validity should be confirmed by further experiments and simulations.

\section{ACKNOWLEDGEMENTS}

This research has been supported by the Czech Science Foundation, grant No. P205/10/0814, LASERLABEUROPE (grant no. 228334), and by the CTU grant SGS10/299/OHK4/3T/14. The work has been done within the activities of the Working Package 10 (Fusion experiment) of the HiPER Project.

\section{REFERENCES}

[1] M. J. Berger, et. al. ESTAR, PSTAR, and ASTAR: computer programs for calculating stopping-power and range tables for electrons, protons, and helium ions. http://physics.nist.gov/Star. Version 1.2.3.
[2] R. Betti, et al. Shock ignition of thermonuclear fuel with high areal density. Phys Rev Lett 98(15):155001, 2007.

[3] R. Cook, et al. Production and characterization of doped mandrels for inertial-confinement fusion experiments. J Vac Sci Technol A 12:1275, 1994.

[4] D. A. Haynes Jr., et al. Chlorine K-shell spectroscopy of directly driven cylindrical implosions. J Quant Spectrosc Radiat Transf 65:297-302, 2000.

[5] G. Holzer, et al. Characterization of flat and bent crystals for X-ray spectroscopy and imaging. Cryst Res Technol 33:555, 1998.

[6] K. Jungwirth, et.al. The Prague Asterix laser system. Phys Plasmas 8(5):2495-2501, 2001.

[7] M. O. Krause. Atomic radiative and radiationless yelds from K and L shells. J Phys Chem Ref Data 8:307, 1979.

[8] J. J. MacFarlane, et.al. SPECT3D - a multi-dimensional collisional-radiative code for generating diagnostic signatures based on hydrodynamics and pic simulation output. High Energy Density Physics 3(1-2):181-190, 2007.

[9] A. Morace, D. Batani. Spherically bent crystal for X-ray imaging of laser produced plasmas. Nucl Instrum Meth A 623:797, 2010.

[10] L. J. Perkins, et al. Shock ignition: A new approach to high gain inertial confinement fusion on the national ignition facility. Phys Rev Lett 103(4):045004, 2009.

[11] S. G Podorov, et al. Optimized polychromatic X-ray imaging with asymmetrically cut bent crystals. J Phys D: Apl Phys 34:2363, 2001. 